\title{
Azacitidine Regimen
}

National Cancer Institute

\section{Source}

National Cancer Institute. Azacitidine Regimen. NCI Thesaurus. Code C159995.

A chemotherapy regimen consisting of azacitidine that may be used in the treatment of myeloproliferative neoplasms, myelodysplastic syndromes, and acute myeloid leukemia (AML). 